\title{
Tensor categories of Coxeter type B and QFT on the half plane
}

Cite as: Journal of Mathematical Physics 38, 5371 (1997); https://doi.org/10.1063/1.531948

Submitted: 31 December 1996 . Accepted: 30 June 1997. Published Online: 04 June 1998

Reinhard Häring-Oldenburg

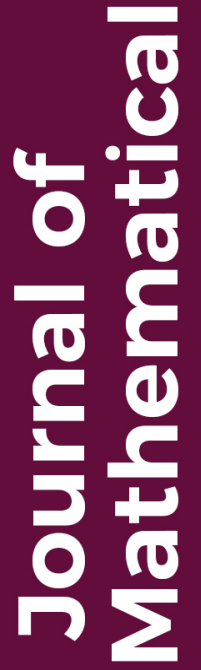

Reinhard Haring-Oldenburg

\section{AVS Quantum Science}

A high impact interdisciplinary journal for ALL quantum science 


\title{
Tensor categories of Coxeter type B and QFT on the half plane
}

\author{
Reinhard Häring-Oldenburga \\ Mathematisches Institut, Bunsenstr. 3-5, 37073 Göttingen, Germany
}

(Received 31 December 1996; accepted for publication 30 June 1997)

We introduce braided tensor categories which are associated to the braid group of Coxeter type B. Connected by a reconstruction theorem there is the notion of B-braided Hopf algebras. These structures show up in quantum field theories defined on the half plane or on the cylinder. (C) 1997 American Institute of Physics. [S0022-2488(97)01010-4]

\section{INTRODUCTION}

Every Coxeter graph defines a braid group that is an infinite covering of its Coxeter group. T. tom Dieck initiated in Refs. 1 and 2 the systematic algebraic study of these braid groups and their quotient algebras for all root systems.

The Coxeter group of A-type is the permutation group and its braid group $\mathrm{ZA}_{n}$ is Artin's braid group. For type $B_{n}$ the Coxeter group is a semidirect product of the permutation group with $\mathbb{Z}_{2}^{n}$.

Definition 1: The braid group $\mathrm{ZB}_{n}$ of Coxeter type $B$ is generated by $\tau_{0}, \tau_{1}, \ldots, \tau_{n-1}$ with relations

$$
\begin{gathered}
\tau_{i} \tau_{j}=\tau_{j} \tau_{i} \quad \text { if }|i-j|>1 \\
\tau_{i} \tau_{j} \tau_{i}=\tau_{i} \tau_{j} \tau_{i} \quad \text { if } i, j \geqslant 1,|i-j|=1 \\
\tau_{0} \tau_{i}=\tau_{i} \tau_{0} \quad \text { if } i \geqslant 2 \\
\tau_{0} \tau_{1} \tau_{0} \tau_{1}=\tau_{1} \tau_{0} \tau_{1} \tau_{0}
\end{gathered}
$$

Generators $\tau_{i}, i \geqslant 1$ satisfy the relations of Artin's braid group.

$\mathrm{ZB}_{n}$ may be graphically interpreted (cf. Fig. 1) as symmetric braids or cylinder braids: The symmetric picture shows it as the group of braids with $2 n$ strands (numbered $-n, \ldots,-1,1, \ldots, n$ ) which are fixed under a 180 degree rotation about the middle axis. In the cylinder picture one adds a single fixed line (indexed 0 ) on the left and obtains $\mathrm{ZB}_{n}$ as the group of braids with $n$ strands that may surround this fixed line. The generators $\tau_{i}, i \geqslant 0$ are mapped to the diagrams $X_{i}^{(G)}$ given in Fig. 1. More generally there are tangles (indicated in Fig. 1 by the TLJ tangles $e_{i}^{(G)}$ ) of B-type that live naturally in a cylinder.

The cylinder interpretation of relation (4) shown in Fig. 2 is the interface to physical applications. One should think of each side of this picture as showing two particles which are reflected on a wall. The equality of both sides expresses the independency of these reflections. The integrability condition for quantum field theories in two dimension is given by the Yang-Baxter equation (YBE) which is a spectral parameter dependend form of (2). If the QFT lives on a half plane with reflecting boundary it is known from the work of Cherednik, Sklyanin, Goshal, and Zamolodchikov (see Ref. 3) that the YBE gets augmented by the reflection equation (or boundary YBE) which is a spectral parameter dependend form of (4). Solutions of the reflection equation can be obtained from tensor representations of quotients of the group algebra of the braid group $\mathrm{ZB}_{n}$ by a Baxterization procedure. ${ }^{4}$ This example inspires our general hypothesis: Whenever

${ }^{a)}$ Electronic mail: haering@cfgauss.uni-math.gwdg.de 


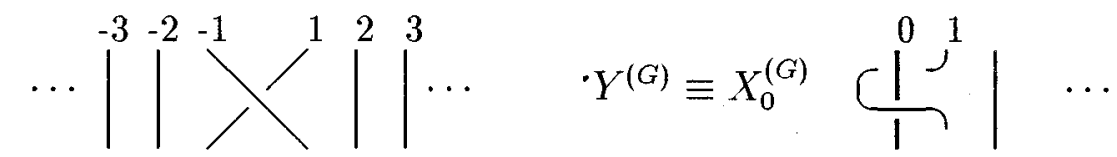

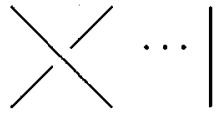

$\left.\left.\left.\left.\cdots\right|^{-3}\right|^{-2}\right|^{-1}\right|^{2} \cdot \cdots$
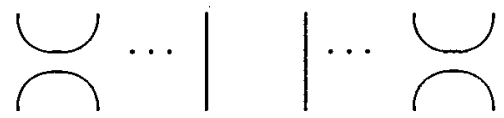

$X_{i}^{(G)}$

$e_{0}^{(G)}$

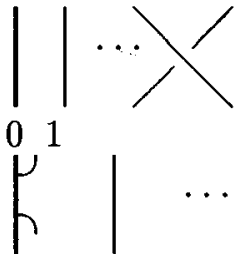

$e_{i}^{(G)}$

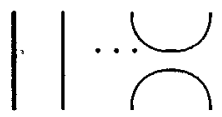

FIG. 1. The graphical interpretation of the generators as symmetric tangles (on the left) and as cylinder tangles (on the right).

Artin's braid group $\mathrm{ZA}_{n}$ occurs in a low dimensional physical model on a space without boundary then the B-type braid group $\mathrm{ZB}_{n}$ occurs if the model is placed on a space with a reflecting boundary. Another illustration of this hypothesis is the fact that the Markov trace on the B-type Temperley-Lieb algebra can be used to express the partition function of a Potts model with boundary interaction. 5

This paper studies the categorical structure underlying these cylinder tangle diagrams. The ultimate goal is to extend the rich theory around ordinary braided tensor categories including quantum groups and knot invariants to the braid group of B type. Some results have already been obtained. Generalizations of Temperley-Lieb algebras, ${ }^{1}$ Hecke algebras, ${ }^{6}$ and Birman-Wenzl algebras ${ }^{7}$ have been studied along with their associated invariants of torus links (which are obtained by closing cylinder braids).

The central observation in the search for the categorial structure of the new type of braiding is the following: The braid generator $\tau_{0}$ (later on $b_{X}$ in the category) does not satisfy the naturality condition with the A-type braiding $\tau_{1}$. Thus, it cannot be a morphisms in a braided tensor
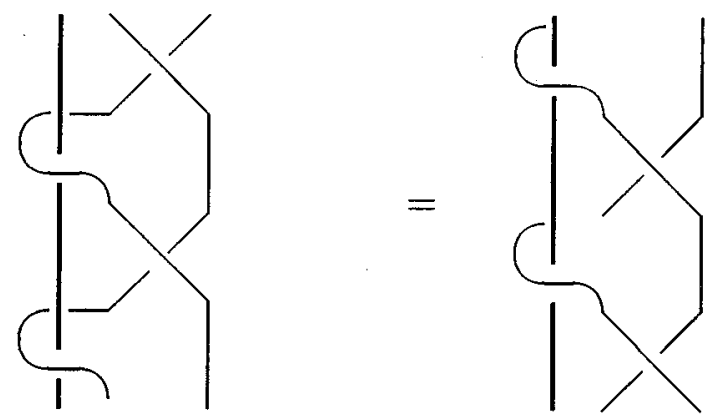

FIG. 2. The cylinder interpretation of relation (4). 
category. We account for this fact by introducing two morphism spaces for every pair of objects in the category. Local morphism that are natural with respect to A-type braidings and global morphisms which need not.

We now outline the structure of the paper. After giving the precise definition the paper introduces the category of amplimorphisms of a Hopf algebra as a first example. The next section introduces Coxeter-B braided Hopf algebras which are related to Coxeter-B categories by a Tannaka-Krein style duality. In the sequel the construction of Coxeter-B braided categories from semi-simple quotients of the group algebra of $\mathrm{ZB}_{n}$ is discussed. The last section is devoted to a physical application. We show that the localized morphisms of an algebraic quantum field theory on the half plane with reflecting boundary form a Coxeter-B braided category.

It is important to observe that the central equation (4) appears also in other contexts. Most important is its fundamental role in Majid's elaborated theory of braided mathematics. ${ }^{8}$ There it appears a commutativity relation between distinct copies of objects that obey braided commutative statistics. Furthermore, the defining relations of Majid's quantum Lie algebras are of the same form. Naturally one should explore the deeper meaning of these connections in further studies. Further topics that have been deferred are the categorial definition of invariants of links in the solid torus (compare Ref. 6), the generalization of evaluation and coevaluation morphisms and the question of invariants of 3-manifolds with boundary.

Preliminaries. We use the language of braided tensor categories (BTC) extensively. Our basic notation follow that of $\mathrm{Majid}^{8}$ and was used already in Ref. 9. The functorial braid isomorphism is denoted $\Psi_{X, Y}: X \otimes Y \neg Y \otimes X X, Y \gtrsim \operatorname{Obj}(\mathscr{C})$. Dual objects are denoted by $X^{*}$ and we use ev and coev for the morphisms of rigidity. We use them to define $\widetilde{q}_{X}:=\left(\mathrm{ev}_{X} \otimes \mathrm{id}_{X^{* *}}\right)^{\circ}\left(\mathrm{id}_{X^{*}} \otimes \Psi_{X^{* *}, X}\right)$ $\circ\left(\operatorname{coev}_{X *} \otimes \operatorname{id}_{X}\right) \in \operatorname{Mor}\left(X, X^{* *}\right)$. There exists a unique morphism $\tau(X) \in \operatorname{Mor}(X, X)$ such that

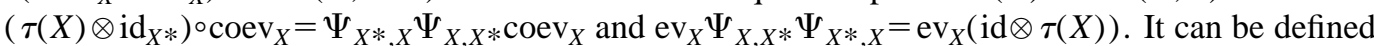
by $\tau\left(X^{*}\right)^{-1}:=\widetilde{q}_{X}^{*} \circ \widetilde{q} X^{*}$. A ribbon category has a natural isomorphism $\sigma(X) \in \operatorname{Mor}(X, X)$ such that $\sigma(X)^{2}=\tau(X), \quad \sigma(X) \otimes \sigma(Y)=\Psi_{Y, X} \Psi_{X, Y} \sigma(X \otimes Y), \quad \sigma\left(X^{*}\right)=\sigma(X)^{*}, \quad f \sigma(X)=\sigma(Y) f \forall f$ $\in \operatorname{Mor}(X, Y)$.

We now give a short review of the reconstruction theorem from Refs. 8, 9. Let $\mathscr{C}$ be a rigid BTC and $F: \mathscr{C} \rightarrow \mathrm{Vec}$ a [(weak) quasi] tensor functor. Then the set $H=\operatorname{Nat}(F, F)$ of natural transformations from $F$ to $F$ carries the structure of a [(weak) quasi] Hopf algebra and there is a functor $G: \mathscr{C} \rightarrow \operatorname{Rep}(H)$ such that $\mathscr{C} \rightarrow \operatorname{Rep}(H) \stackrel{V}{\rightarrow}$ Vec composes to $F$. In the case of a faithful functor and a semisimple category, $\mathscr{C}$ and $\operatorname{Rep}(H)$ are equivalent BTCs.

$$
\begin{gathered}
H:=\operatorname{Nat}(F, F)=\left\{h: \operatorname{Obj}(\mathscr{C}) \rightarrow \operatorname{End}_{\mathrm{Vec}} \mid h_{X} \in \operatorname{End}(F(X)),\right. \\
\left.F(f) \circ h_{X}=h_{Y} \circ F(f) \forall X, Y \in \operatorname{Obj}(\mathscr{C}) \forall f \in \operatorname{Mor}(X, Y)\right\} .
\end{gathered}
$$

$H$ is a vector space by pointwise addition. The multiplication is also defined pointwise: $(h g)_{X}$ : $=h_{X^{\circ}} g_{X} X \in \operatorname{Obj}(\mathscr{C}), h, g \in H$. The unit is $X \mapsto 1_{X}=\mathrm{id}_{F(X)}$. The coproduct $\Delta: H \rightarrow H \otimes H$ is defined

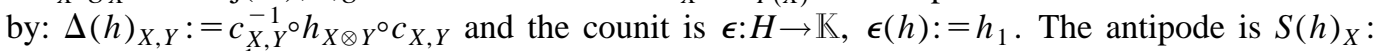
$=d_{X}^{*}\left(h_{X^{*}}\right)^{*} d_{X}^{*-1}$, where the isomorphisms $d_{X}: F(X)^{*} \rightarrow F\left(X^{*}\right)$ with the property $d_{X^{\circ}} F(f)^{*}=F\left(f^{*}\right)^{\circ} d_{Y} \forall f \in \operatorname{Mor}(X, Y)$ exists by definition of a [(weak) quasi] tensor functor. (See Ref. 9 for more details and the construction of such functors.) If $\mathscr{C}$ is a ribbon category then there is a ribbon element $v_{X}:=F(\sigma(X))$ in $H$. The vector spaces $F(X)$ are representation spaces of $H$. The representations are $\varrho_{X}(h) \cdot v:=h_{X}(v) h \in H, \quad v \in F(X)$. This induces a functor $G: \mathscr{C} \rightarrow \operatorname{Rep}(H)$.

\section{COXETER-B BRAIDED CATEGORIES}

As mentioned in Sec. I, the surrounding of the cylinder axis (generator $\tau_{0}$ in the braid group $\mathrm{ZB}_{n}$ ) does not behave natural with respect to the braiding $\tau_{1}$. Therefore, it can not be represented by a morphism in a BTC. Nevertheless, we can incorporate it by defining a bigger space of 
morphisms that are not bound to satisfy naturality with the ordinary braiding. This seems natural from the physical considerations that we undertake in the last section. From a purely mathematical point of view one should probably prefer to view B-type tangles as a kind of module for an ordinary tangle category. ${ }^{10}$

Definition 2: Let $\mathscr{C}$ be a rigid BTC. A Coxeter-B braided category over $\mathscr{C}$ is an embedding of $\mathscr{C}$ in a rigid monoidal category $\hat{C}$ such that the following list of axioms holds. Morphisms $\operatorname{Mor}(X, Y):=\operatorname{Mor}_{\mathscr{C}}(X, Y)$ are said to be local and morphisms $\operatorname{Mor}^{(G)}(X, Y):=\operatorname{Mor}_{\mathscr{C}}(X, Y)$ are said to be global.

$$
\begin{gathered}
\operatorname{Obj}(\mathscr{C})=\operatorname{Obj}(\hat{\mathscr{C}}), \\
\exists i: \operatorname{Mor}(X, Y) \rightarrow \operatorname{Mor}^{(G)}(X, Y) \quad \text { monomorphism, } \\
\forall X \exists b_{X} \in \operatorname{Mor}^{(G)}(X, X) \quad \text { invertible, } \\
b_{Y} f=f b_{X} \quad \forall f \in \operatorname{Mor}(X, Y), \\
\pi\left(b_{X}\right)=\mathrm{id}_{X} \quad b_{1}=\mathrm{id}_{1}, \\
\operatorname{id}_{X} \otimes b_{Y}=\Psi_{Y, X}\left(b_{Y} \otimes \mathrm{id}_{X}\right) \Psi_{X, Y}, \\
b_{X} \otimes b_{Y}=b_{X \otimes Y} \Omega_{Y, X} \Psi_{X, Y}=\Psi_{Y, X} \Psi_{X, Y} b_{X \otimes Y}, \\
b_{X}^{*}=\sigma\left(X^{*}\right)^{2} b_{X^{*}}^{-1} .
\end{gathered}
$$

We say that $\hat{\mathscr{C}}$ has a projection if we have in addition:

$$
\begin{gathered}
\exists \pi: \operatorname{Mor}^{(G)}(X, Y) \rightarrow \operatorname{Mor}(X, Y) \quad \text { epimorphism, } \\
\pi \circ i=\mathrm{id}, \\
\pi(f \otimes g)=\pi(f) \otimes g \quad \text { if } g \in \operatorname{Mor}(X, Y) .
\end{gathered}
$$

The axioms ensure that the two categories are almost equal. They differ only by the existence of some global morphisms. If needed, one may restrict this extension to a minimum by postulating

$$
\operatorname{Mor}^{(G)}(X, Y)=\left\{b_{Y}^{n} \mid n \in \mathbb{Z}\right\} \circ \operatorname{Mor}(X, Y) \in\left\{b_{X}^{n} \mid n \in \mathbb{Z}\right\} .
$$

Note that $\Psi$ is a braiding of $\mathscr{C}$, not of $\hat{\mathscr{C}}$. This makes (10) possible which otherwise would give a contradiction to naturality of $\Psi$.

The graphical idea behind the projection is to simply forget about the cylinder axis.

In some applications it may be more natural to work with $b_{X}^{\prime}:=\sigma(X)^{-1} b_{X}$ which fulfills $b_{X \otimes Y}^{\prime}=\left(\sigma(X)^{-1} \otimes \sigma(Y)^{-1}\right) \Psi^{2}\left(b_{X} \otimes b_{Y}\right) \Psi^{-2}=b_{X}^{\prime} \otimes b_{Y}^{\prime} \quad$ and $\quad b_{X}^{\prime *}=b_{X}^{*} \sigma(X)^{-1 *}=\sigma\left(X^{*}\right)^{2}$ $\times b_{X^{*}}^{-1} \sigma\left(X^{*}\right)^{-1}=\left(b_{X^{*}} \sigma\left(X^{*}\right)^{-1}\right)^{-1}=\left(b_{X^{*}}^{\prime}\right)^{-1}$.

Lemma 1:

$$
\begin{gathered}
\pi\left(\mathrm{id}_{X} \otimes b_{Y}\right)=\Psi_{Y, X} \Psi_{X, Y}, \\
\Psi_{Y, X}\left(b_{Y} \otimes \mathrm{id}_{X}\right) \Psi_{X, Y}\left(b_{X} \otimes \mathrm{id}_{Y}\right)=\left(b_{X} \otimes \mathrm{id}_{Y}\right) \Psi_{Y, X}\left(b_{Y} \otimes \mathrm{id}_{X}\right) \Psi_{X, Y}, \\
\operatorname{ev}_{X}\left(b_{X^{*}} \otimes \mathrm{id}_{X}\right) \Psi_{X, X} *\left(b_{X} \otimes \mathrm{id}_{X^{*}}\right)=\operatorname{ev}_{X} \Psi_{X, X} *
\end{gathered}
$$




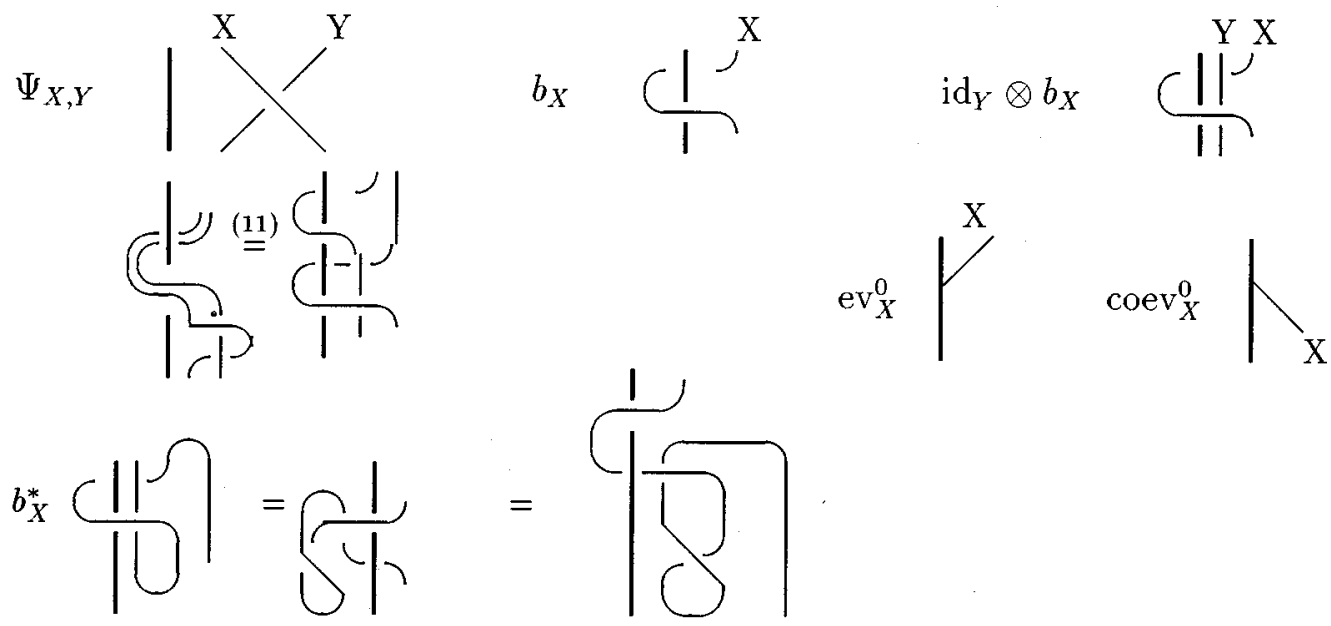

FIG. 3. Graphical calculus for Coxeter-B braided categories.

Proof: (18) is trivial and (19) is simply commutativity of $b_{X} \otimes \mathrm{id}_{Y}$ and $\operatorname{id}_{X} \otimes b_{Y}$. We now prove (20)

$$
\begin{aligned}
\operatorname{ev}_{X}\left(b_{X^{*}} \otimes \operatorname{id}_{X}\right) \Psi_{X, X^{*}}\left(b_{X} \otimes \operatorname{id}_{X^{*}}\right) & =\operatorname{ev}_{X}\left(b_{X^{*}} \otimes \operatorname{id}_{X}\right) \Psi_{X, X^{*}}\left(b_{X} \otimes \operatorname{id}_{X^{*}}\right) \Psi_{X^{*}, X} \Psi_{X^{*}, X}^{-1} \\
& =\operatorname{ev}_{X}\left(b_{X^{*}} \otimes b_{X}\right) \Psi_{X^{*}, X}^{-1} \\
& =\operatorname{ev}_{X} b_{X^{*} \otimes X} \Psi_{X, X^{*}} \Psi_{X^{*}, X} \Psi_{X^{*}, X}^{-1} \\
& =b_{1} \mathrm{ev}_{X} \Psi_{X, X^{*}} \\
& =\operatorname{ev}_{X} \Psi_{X, X^{*}} .
\end{aligned}
$$

This structure can be incorporated into the graphical calculus for tensor categories (see, e.g., Ref. 8) by extending every diagram by a fixed line on the left and representing $b$ by surrounding this line as indicated in Fig. 3. The tensor product $f \otimes g$ of two global morphisms is obtained by replacing the fixed line of $g$ by the whole graph of $f$. The graphical interpretation makes (10) obvious. To understand the graphical origin of (12) note that $b_{X}^{*}=\left(\mathrm{ev}_{X} \otimes \mathrm{id}\right)\left(\mathrm{id} \otimes b_{X} \otimes \mathrm{id}\right)$ $\times\left(\operatorname{id} \otimes \operatorname{coev}_{X}\right)$. It is shown in the last line of Fig. 3 that this is graphically equivalent to $\left(\mathrm{ev}_{X} \otimes \mathrm{id}\right)\left(\Psi_{X, X^{*}} \Psi_{X^{*}, X} \otimes \mathrm{id}\right)\left(\mathrm{id} \otimes \operatorname{coev}_{X}\right) b_{X^{*}}^{-1}=\left(\mathrm{ev}_{X} \otimes \mathrm{id}\right)(\mathrm{id} \otimes \tau(X) \otimes \mathrm{id})\left(\mathrm{id} \otimes \operatorname{coev}_{X}\right) b_{X^{*}}^{-1}$ $=\tau(X)^{*} b_{X^{*}}^{-1}=\tau\left(X^{*}\right) b_{X^{*}}^{-1}=\sigma\left(X^{*}\right)^{2} b_{X^{*}}^{-1}$

Note that the whole construction of Coxeter-B braided BTC was guided by the wish to have for every $X \in \operatorname{Obj}(\hat{\mathscr{C}}) \quad$ a morphism $\quad \mathrm{ZB}_{n} \rightarrow \operatorname{End}\left(X^{\otimes n}\right) \quad$ mapping $\tau_{0} \mapsto b_{X} \otimes \mathrm{id}_{X}^{\otimes(n-1)} \quad$ and $\tau_{i} \mapsto \mathrm{id}_{X}^{\otimes(i-1)} \otimes \Psi_{X, X} \otimes \mathrm{id}_{X}^{\otimes(n+1-i)}$.

In this paper we use the above definition but we note that there are applications where it is convenient to relax some of the axioms or add new ones. One way to relax the axioms is to use $b_{X}^{\prime}$ as the fundamental global morphism and replace (11) and (10) by $b_{X \otimes Y}^{\prime}=\Psi_{Y, X}\left(b_{Y}^{\prime}\right.$ $\left.\otimes \operatorname{id}_{X}\right) \Psi_{X, Y}\left(b_{X}^{\prime} \otimes \operatorname{id}_{Y}\right)=\left(b_{X}^{\prime} \otimes \operatorname{id}_{Y}\right) \Psi_{Y, X}\left(b_{Y}^{\prime} \otimes \operatorname{id}_{X}\right) \Psi_{X, Y}$. This defines what we call a restricted Coxeter-B braided category.

It is possible to define a B-type analog of rigidity. We say that a Coxeter-B braided category $\hat{\mathscr{C}}$ is B-rigid if there are global morphisms $\operatorname{ev}_{X}^{0} \in \operatorname{Mor}^{(G)}(X, 1), \operatorname{coev}_{X}^{0} \in \operatorname{Mor}^{(G)}(1, X)$ such that $\pi\left(\mathrm{ev}^{0}\right)=\pi\left(\operatorname{coev}^{0}\right)=0$. One may also wish to postulate $\left(\mathrm{ev}_{X}^{0} \otimes \mathrm{id}_{X^{*}}\right) \operatorname{coev}_{X}=\operatorname{coev}_{X^{*}}^{0}$ and 
$\mathrm{ev}_{X}\left(\operatorname{coev}_{X^{*}}^{0} \otimes \mathrm{id}_{X}\right)=\mathrm{ev}_{X}^{0}$. Furthermore, one may wish to demand $\mathrm{ev}_{X}^{0} \operatorname{coev}_{X}^{0}=\mathrm{id}_{1}$. A graphical representation of these global morphisms are given in Fig. 3. However, there is no obvious way to draw a picture for $\operatorname{id}_{X} \mathrm{ev}_{Y}^{0}$. One would need an analog of (10). Therefore, one may have to give up the assumption that $\hat{\mathscr{C}}$ is a monoidal category in situations where the graphical calculus of ev ${ }^{0}$ is essential. Such a situation is studied in detail in Ref. 10.

\section{AMPLIMORPHISMS}

As a first example we study amplimorphisms. Let $H=H(m, 1, \Delta, \epsilon, S, R, v)$ be a quasitriangular ribbon Hopf algebra. An amplimorphism of $H$ is a monomorphism $\chi: H \rightarrow H \otimes \operatorname{End}_{\mathrm{Vec}}\left(V_{\chi}\right)$, where $V_{\chi}$ is some vector space. The category $\operatorname{Amp}(H)$ of amplimorphisms of $H$ has as objects the amplimorphisms of $A$ and as morphisms the sets $\operatorname{Mor}_{\text {Amp }}\left(\chi_{1}, \chi_{2}\right):=\{T \in H$ $\left.\otimes \operatorname{Mor}_{\mathrm{Vec}}\left(V_{\chi_{1}}, V_{\chi_{2}}\right) \mid T \chi_{1}(a)=\chi_{2} T(a) \forall a \in H\right\}$. The monoidal structure is given on objects by $\chi_{1} \otimes \chi_{2}:=\left(\chi_{1} \otimes \mathrm{id}\right) \chi_{2}$ and on morphisms by $T_{1} \otimes T_{2}:=\left(T_{1} \otimes \mathrm{id}\right)\left(\chi_{1} \otimes \mathrm{id}\right)\left(T_{2}\right)$, where $\chi_{1}$ is the source of $T_{1}$.

The counit of $H$ gives rise to a functor $D: \operatorname{Amp}(H) \rightarrow \operatorname{Rep}(H)$ with $D(\chi)(a):=(\epsilon$ $\otimes$ id $) \chi(a), D(T):=(\epsilon \otimes \mathrm{id})(T)$ and the coproduct induces $A: \operatorname{Rep}(H) \rightarrow \operatorname{Amp}(H)$ given by $A(\varrho)$ $\times(a):=(\operatorname{id} \otimes \varrho)(\Delta(a)), A(T):=1 \otimes T$. Obviously one has $D A=\operatorname{Id}$ and $A\left(\varrho_{1}\right) \otimes A\left(\varrho_{2}\right)=($ id $\left.\otimes \varrho_{1} \otimes \varrho_{2}\right)((\Delta \otimes \mathrm{id}) \Delta)=A\left(\varrho_{1} \otimes \varrho_{2}\right)$.

Define RAmp $(H)$ to be the full closure of $A(\operatorname{Rep}(H))$.

Proposition 2: $\operatorname{RAmp}(H)$ is a Coxeter-B braided category with projection over $\operatorname{Rep}(H)$.

Proof: Inclusion and projection morphisms $i, \pi$ are induced by $A, D$. The global braid morphisms $b$ on an object $\chi=A(\varrho)$ is given by

$$
b_{\chi}:=(\mathrm{id} \otimes \varrho)\left(R_{2,1} R\right) .
$$

This is a morphism of RAmp because $\chi(a) b_{\chi}=(\mathrm{id} \otimes \varrho)(\Delta(a))(\mathrm{id} \otimes \varrho)\left(R_{2,1} R\right)=(\operatorname{id} \otimes \varrho)$ $\times\left(\Delta(a) R_{2,1} R\right)=(\mathrm{id} \otimes \varrho)\left(R_{2,1} R \Delta(a)\right)=b_{\chi} \chi(a)$. Note that $D\left(b_{\chi}\right)=\mathrm{id}$ and thus $\pi\left(b_{\chi}\right)=\mathrm{id}$.

We show (10) for amplimorphisms $\chi_{i}=A\left(\varrho_{i}\right)$ in the following calculation

$$
\begin{aligned}
& \Psi_{\chi_{2}, \chi_{1}}\left(b_{\chi_{2}} \otimes \mathrm{id}\right) \Psi_{\chi_{1}, \chi_{2}} \\
& \quad=\left(\mathrm{id} \otimes\left(\Psi^{\mathrm{Vec}}\left(\varrho_{2} \otimes \varrho_{1}\right)(R)\right)\right)\left(\mathrm{id} \otimes \varrho_{2} \otimes \mathrm{id}\right)\left(R_{2,1} R \otimes 1\right)\left(\mathrm{id} \otimes\left(\Psi^{\mathrm{Vec}}\left(\varrho_{1} \otimes \varrho_{2}\right)(R)\right)\right) \\
& \quad=\left(\mathrm{id} \otimes \varrho_{1} \otimes \varrho_{2}\right)\left(R_{3,2} R_{3,1} R_{1,3} R_{2,3}\right) \\
& \quad=\left(\mathrm{id} \otimes \varrho_{1} \otimes \varrho_{2}\right)\left((\Delta \otimes \mathrm{id})\left(R_{2,1} R\right)\right) \\
& \quad=\left(\mathrm{id} \otimes \varrho_{1} \otimes \mathrm{id}\right)(\Delta \otimes \mathrm{id})\left(\mathrm{id} \otimes \varrho_{2}\right)\left(R_{2,1} R\right) \\
& \quad=\left(\chi_{1} \otimes \mathrm{id}\right)\left(b_{\chi_{2}}\right)=\mathrm{id}_{\chi_{1}} \otimes b_{\chi_{2}} .
\end{aligned}
$$

Using this we prove (11)

$$
\begin{aligned}
b_{\chi_{1}} \otimes b_{\chi_{2}} & =\left(b_{\chi_{1}} \otimes \mathrm{id}\right)\left(\mathrm{id} \otimes b_{\chi_{2}}\right) \\
& =\left(\mathrm{id} \otimes \varrho_{1} \otimes \varrho_{2}\right)\left(\left(R_{2,1} R\right)\left(R_{3,2} R_{3,1} R_{1,3} R_{2,3}\right)\right) \\
& =\left(\mathrm{id} \otimes \varrho_{1} \otimes \varrho_{2}\right)\left(R_{2,1} R_{3,1} R_{3,2} R_{1,2} R_{1,3} R_{2,3}\right) \\
& =\left(\mathrm{id} \otimes \varrho_{1} \otimes \varrho_{2}\right)\left(R_{2,1} R_{3,1} R_{1,3} R_{1,2} R_{3,2} R_{2,3}\right) \\
& =\left(\mathrm{id} \otimes \varrho_{1} \otimes \varrho_{2}\right)(\mathrm{id} \otimes \Delta)\left(R_{2,1} R\right)\left(\mathrm{id} \otimes \varrho_{1} \otimes \varrho_{2}\right)\left(1 \otimes R_{2,1} R\right) \\
& =b_{\chi_{1} \otimes \chi_{2}} \Psi_{\varrho_{2}, \varrho_{1}} \Psi_{\varrho_{1}, \varrho_{2}} .
\end{aligned}
$$


Equation (12) may also be verified directly. The proof consists of simply repeating the arguments given above in justifying axiom (12) from its graphical interpretation.

A similar use of the quantum group element $R_{2,1} R$ is made in Ref. 11 (compare also its role in the theory of quantum Lie algebras ${ }^{8}$ ) where generalized tensor representations of the braid group $\mathrm{ZB}_{n}$ are constructed.

\section{COXETER-B BRAIDED HOPF ALGEBRAS}

In this section we define generalizations of Hopf algebras that are conected to B-type BTCs by a Tannaka-Krein style duality.

Definition 3: A [(weak) quasi] Coxeter-B braided Hopf algebra H is a [(weak) quasi] quasitriangular ribbon Hopf algebra with an element $\bar{v} \in H$ such that

$$
\begin{gathered}
1 \otimes \bar{v}=R_{2,1}(1 \otimes \bar{v}) R, \\
\epsilon(\bar{v})=1, \\
\Delta(\bar{v})=(\bar{v} \otimes \bar{v})\left(R_{21} R\right)^{-1}=\left(R_{21} R\right)^{-1}(\bar{v} \otimes \bar{v}), \\
S(\bar{v})=v^{2} \bar{v}^{-1} .
\end{gathered}
$$

Definition 4: A restricted Coxeter-B braided [(weak) quasi] Hopf algebra $H$ is a [(weak) quasi] quasitriangular ribbon Hopf algebra with an element $\bar{v} \in H$ such that

$$
\begin{gathered}
R_{2,1} \bar{v}_{2} R \bar{v}_{1}=\bar{v}_{1} R_{2,1} \bar{v}_{2} R, \\
\epsilon(\bar{v})=1, \\
\Delta(\bar{v})=R^{-1}(1 \otimes \bar{v}) R(\bar{v} \otimes 1), \\
S(\bar{v})^{-1}=S(u)^{-1} \sum_{j} \alpha_{j} \bar{v} S\left(\beta_{j}\right) .
\end{gathered}
$$

Here, we have used $R=\Sigma_{j} \alpha_{j} \otimes \beta_{j}, u:=\Sigma_{j} S\left(\beta_{j}\right)=\alpha_{j}$.

The restricted case is really a restriction: (26) is obvious and (28) is $\Delta(\vec{v})=\left(R_{2,1} R\right)^{-1}$ $(\bar{v} \otimes \bar{v})=R^{-1} R_{2,1}^{-1} R_{2,1}(1 \otimes \bar{v}) R(\bar{v} \otimes 1)=R^{-1} \bar{v}_{2} R \bar{v}_{1}$. Equation (29) follows from the coproduct and counit formulas

$$
\begin{gathered}
1=\epsilon(\bar{v})=m(S \otimes \mathrm{id}) \Delta(\bar{v})=\sum_{i, j} m(S \otimes \mathrm{id})\left(S\left(\alpha_{i}\right) \alpha_{j} \bar{v} \otimes \beta_{i} \bar{v} \beta_{j}\right)=\sum_{i, j} S(\bar{v}) S\left(\alpha_{j}\right) S^{2}\left(\alpha_{i}\right) \beta_{i} \bar{v} \beta_{j}, \\
S(\bar{v})^{-1}=\sum_{j} S\left(\alpha_{j}\right) S\left(u^{-1}\right) \bar{v} \beta_{j}=\sum_{j} C^{-1} S\left(\alpha_{j}\right) u \bar{v} \beta_{j}=\sum_{j} C^{-1} S^{2}\left(\alpha_{j}\right) u \bar{v} S\left(\beta_{j}\right) \\
=C^{-1} u \sum_{j} \alpha_{j} \bar{v} S\left(\beta_{j}\right) .
\end{gathered}
$$

The calculations use the facts that $R^{-1}=(S \otimes \mathrm{id})(R), R=(S \otimes S)(R), u^{-1}=\Sigma_{j} \beta_{j} S^{2}\left(\alpha_{j}\right), C$ : $=u S(u)$ central. In the nonrestricted case (29) can be further simplified to obtain (25) by using the relation obtained from the application of $m(S \otimes \mathrm{id})$ to (22).

We have the following Tannaka-Krein style duality between Coxeter B-braided Hopf algebras and B-type tensor categories. 
Proposition 3: The representation category $\operatorname{Rep}(H)$ of a Coxeter-B braided [(weak) quasi] Hopf algebra algebra is a B-braided tensor category.

Proof: Let $\mathscr{C}$ be the standard representation category of $H$ as a [(weak) quasi] Hopf algebra (Ref. 9). For an object $X$ which is a representation $\varrho_{X}: H \rightarrow \operatorname{End}\left(V_{X}\right)$ one sets $b_{X}:=\varrho_{X}(\bar{v})$. Equation (8) is then trivial. The second equation from (9) follows from (23). Global morphism spaces are defined by (17).

Applying a tensor product representation $\varrho_{X} \otimes \varrho_{Y}$ to (22) implies (10): $\operatorname{id}_{X} \otimes b_{Y}=\left(\varrho_{X} \otimes \varrho_{Y}\right)$ $\left(R_{2,1}\right)\left(\mathrm{id}_{X} \otimes b_{Y}\right)\left(\varrho_{X} \otimes \varrho_{Y}\right)(R)=\Psi_{\varrho_{Y}, \varrho_{X}}\left(b_{Y} \otimes \mathrm{id}_{X}\right) \Psi_{\varrho_{X}, \varrho_{Y}}$. In a similar way one proves (11) from (24): $\quad b_{X} \otimes b_{Y}=b_{X \otimes Y} \Psi_{Y, X} \Psi_{X, Y} \Leftrightarrow \varrho_{X}(\bar{v}) \otimes \varrho_{Y}(\bar{v})=\left(\varrho_{X} \otimes \varrho_{Y}\right)\left(\Delta(\bar{v}) R_{2,1} R\right)=\left(\varrho_{X} \otimes \varrho_{Y}\right)$ $(\Delta(\bar{v}))\left(\varrho_{X} \otimes \varrho_{Y}\right)\left(R_{2,1}\right) \Psi_{V_{Y}, V_{X}}^{\mathrm{Vec}} \Psi_{V_{X}, V_{Y}}^{\mathrm{Vec}}\left(\varrho_{X} \otimes \varrho_{Y}\right)(R)=b_{X \otimes Y} \Psi_{Y, X} \Psi_{X, Y}$.

Equation (12) is implied by (25): $b_{X *}=\varrho_{X}(S(\bar{v}))^{*}=\varrho_{X}\left(v^{2} \bar{v}^{-1}\right)^{*}=\varrho_{X}\left(v^{2}\right)^{*} b_{X}^{*-1}$ $=\sigma\left(X^{*}\right)^{2} b_{X}^{*-1}$

Restricted Coxeter-B braided Hopf algebras lead only to restricted Coxeter-B braided categories.

Proposition 4: If $\hat{\mathscr{C}}$ is a Coxeter-B braided tensor category and $F: \hat{\mathscr{C}} \rightarrow \mathrm{Vec}$ is a [(weak) quasi] tensor functor, then the set of natural transformations from $F_{\mid C}$ to itself

$$
\begin{gathered}
H:=\left\{h: \operatorname{Obj}(\mathscr{C}) \rightarrow \operatorname{End}_{\mathrm{Vec}} \mid h_{X} \in \operatorname{End}(F(X)),\right. \\
\left.F(f) \circ h_{X}=h_{Y} \circ(f) \forall X, Y \in \operatorname{Obj}(\mathscr{C}) \forall f \in \operatorname{Mor}(X, Y)\right\},
\end{gathered}
$$

carries the structure of a Coxeter-B braided [(weak) quasi] Hopf algebra. If $F$ is a tensor functor on $\hat{\mathscr{C}}$ only in the restricted sense that the naturality equation $c_{X, Y}(F(f) \otimes F(g))=F(f \otimes g) c_{X, Y}$ holds only if $g$ is a local morphism then $H$ is a restricted Coxeter-B braided [(weak) quasi] Hopf algebra.

Proof: This proposition is a corollary to the reconstruction theorem given in Ref. 9 . The only thing we have to check here is that we have a suitable element $\bar{v} \in H$. We set $\bar{v}_{X}:=F\left(b_{X}\right)$. We have $\bar{v} \in H$ because $\forall f \in \operatorname{Mor}(X, Y) F(f) \bar{v}_{X}=\bar{v}_{Y} F(f) \Leftrightarrow F\left(f b_{X}\right)=F\left(b_{Y} f\right)$ and this is true because of naturality of $b$ with respect to local morphisms. In general, $\bar{v}_{X}$ will not be central. Equation (23) is equivalent to (9): $\epsilon(\bar{v})=\bar{v}_{1}=F\left(b_{1}\right)=F\left(\mathrm{id}_{1}\right)=1$.

We first consider the case that $F$ is a tensor functor for all morphisms. We apply $F$ to (10), express $F\left(\Psi_{X, Y}\right)=c \Psi^{\mathrm{Vec}} R_{X, Y} c^{-1}$ and multiply from the left with $c^{-1}$ and from the right with $c$. Then we obtain $c^{-1} F\left(\mathrm{id}_{X} \otimes b_{Y}\right) c=\Psi^{\mathrm{Vec}} R c^{-1} F\left(b_{Y} \otimes \mathrm{id}\right) c \Psi^{\mathrm{Vec}} R \Leftrightarrow 1 \otimes F\left(b_{Y}\right)=\Psi^{\mathrm{Vec}} R$ $\times\left(F\left(b_{Y}\right) \otimes 1\right) \Psi^{\mathrm{Vec}} R \Leftrightarrow(22)$. We show (24):

$$
\begin{aligned}
\Delta(\bar{v})_{X, Y} & =c_{X, Y}^{-1} \circ F\left(b_{X \otimes Y}\right) \circ c_{X, Y}=c_{X, Y}^{-1} \circ F\left(\Psi_{X, Y}^{-1}\right) \circ c_{Y, X} \circ c_{Y, X}^{-1} \circ F\left(\Psi_{Y, X}^{-1}\right) \circ c_{X, Y} \circ c_{X, Y}^{-1} \circ F\left(b_{X} \otimes b_{Y}\right) \circ c_{X, Y} \\
& =\left(\left(R_{2,1} R\right)^{-1}\right)_{X, Y} \circ\left(F\left(b_{X}\right) \otimes F\left(b_{Y}\right)\right)=\left(\left(R_{2,1} R\right)^{-1}(\bar{v} \otimes \bar{v})\right)_{X, Y} .
\end{aligned}
$$

We prove (25) by a simple calculation:

$$
\begin{aligned}
S(\vec{v})_{X} & =d_{X}^{*} \circ\left(\bar{v}_{X *}\right)^{* \circ} d_{X}^{*-1}=d_{X}^{* \circ} F\left(b_{X *}\right)^{* \circ} d_{X}^{*-1}=d_{X}^{* \circ} F\left(b_{X}^{*-1} \sigma(X)^{* 2}\right)^{* \circ} d_{X}^{*-1} \\
& =d_{X}^{*} F\left(\sigma(X)^{*}\right)^{*} d_{X}^{*-1} d_{X}^{*} F\left(\sigma(X)^{*}\right)^{*} d_{X}^{*-1} d_{X}^{*} F\left(b_{X}^{-1 *}\right)^{*} d_{X}^{*-1} \\
& =S(v)_{X} S(v)_{X} F\left(b_{X}^{-1}\right)=\left(v^{2} \bar{v}^{-1}\right)_{X} .
\end{aligned}
$$

Now, we consider the case that $F$ is only a tensor functor in the restricted sense. Then the above proof of (22) and thus also the proof of (24) break down. Equation (26) is shown by applying $F$ to (19) and expressing $F\left(\Psi_{X, Y}\right)=c \Psi^{\mathrm{Vec}} R_{X, Y} c^{-1}$. To show (28) we note that $b_{X \otimes Y}$ $=\Psi^{-2} \Psi_{Y, X}\left(b_{Y} \otimes \mathrm{id}_{X}\right) \Psi_{X, Y}\left(b_{X} \otimes \mathrm{id}_{Y}\right)=\Psi_{X, Y}^{-1}\left(b_{Y} \otimes \mathrm{id}_{X}\right) \Psi_{X, Y}\left(b_{X} \otimes \mathrm{id}_{Y}\right)$. We apply $F$ and multiply from the left with $c^{-1}$ and from the right with $c$ to obtain at the left-hand side $c_{X, Y}^{-1} F\left(b_{X \otimes Y}\right)$ $\times c_{X, Y}=\Delta(\bar{v})_{X, Y}$ and at the right-hand side $c_{X, Y}^{-1} F\left(\Psi_{X, Y}^{-1}\right) F\left(b_{Y} \otimes \operatorname{id}_{X}\right) F\left(\Psi_{X, Y}\right) F\left(b_{X} \otimes \operatorname{id}_{Y}\right) c_{X, Y}$ 
$=R_{X, Y}^{-1} \Psi^{\mathrm{Vec}} c_{Y, X}^{-1} F\left(b_{Y} \otimes \mathrm{id}_{X}\right) F\left(\Psi_{X, Y}\right) c_{X, Y}\left(F\left(b_{X}\right) \otimes \mathrm{id}\right)=R_{X, Y}^{-1} \Psi^{\mathrm{Vec}}\left(F\left(b_{Y}\right) \otimes \mathrm{id}\right) c_{Y, X}^{-1} F\left(\Psi_{X, Y}\right) c_{X, Y}$ $\times\left(F\left(b_{X}\right) \otimes \mathrm{id}\right)=R_{X, Y}^{-1} \Psi^{\mathrm{Vec}}\left(F\left(b_{Y}\right) \otimes \mathrm{id}\right) \Psi^{\mathrm{Vec}} R_{X, Y}\left(F\left(b_{X}\right) \otimes \mathrm{id}\right)=\left(R^{-1}(1 \otimes \bar{v}) R(\bar{v} \otimes 1)\right)_{X, Y}$.

Despite the reconstruction theorem we do not yet have examples of Coxeter-B braided Hopf algebras. The amplimorphisms form a Coxeter-B braided category. But we have no (weak) tensor functor that is also defined on the $b_{X}$ morphism. The next section will present a Coxeter-B braided category for which a restricted tensor functor is known. In this way we prove the existence of restricted Coxeter-B braided Hopf algebras in an indirect manner. Moreover, one can derive an explicit formula for $\bar{v}$ in the quantum Weyl group (we use notation from 12).

Proposition 5: The quantum Weyl Group of $\mathrm{sl}_{2}$ is a restricted Coxeter-B braided Hopf algebra

$$
\bar{v}=w q^{-H^{2} / 8} \sum_{m=0}^{\infty} \beta_{m} q^{-H m / 4} Y^{m},
$$

where $\beta_{0}=1, \beta_{1}$ is arbitrary and

$$
\beta_{a+1}=\left(\beta_{a} \beta_{1}+\beta_{a-1}\left(q^{-1}-1\right) q^{(1-a) / 2}\right) /[a+1] .
$$

For a proof of this proposition see Ref. 13.

There is another approach that assigns an algebra to the category RAmp $(H)$. In Ref. 9 we have introduced the notion of ultra weak Hopf algebras and shown that they can be constructed from ultra weak tensor functors. Such functors need not obey $F(1)=1$. Let $\chi=A(\varrho)$ be the amplimorphism of a $H$ representation $\varrho: H \rightarrow \operatorname{End}(V)$. Then we may set $F(\chi):=H \otimes V$. This defines an ultra weak tensor functor $F: \operatorname{RAmp}(H) \rightarrow \operatorname{Vec}$. As required for ultra weak tensor functors there are functorial epimorphisms $c_{\chi_{1}, \chi_{2}}: F\left(\chi_{1}\right) \otimes F\left(\chi_{2}\right) \rightarrow F\left(\chi_{1} \otimes \chi_{2}\right)$. They are given by $c_{\chi_{1}, \chi_{2}}:=\left(m \otimes \mathrm{id}_{V_{1}} \otimes \mathrm{id}_{V_{2}}\right)\left(\mathrm{id}_{H} \otimes \Psi_{V_{1}, H}^{\mathrm{Vec}} \otimes \mathrm{id}_{V_{2}}\right)$. Here $m: H \otimes H \rightarrow H$ is the multiplication map. The reconstruction from this functor gives an ultra weak Coxeter-B braided Hopf algebra.

The existence of nonrestricted B-braided Hopf algebras remain an open question.

\section{CONSTRUCTION OF COXETER-B BRAIDED TENSOR CATEGORIES}

Coxeter-B braided categories can be obtained from suitable quotients of the braid group $\mathrm{ZB}_{n}$ in the same way that BTCs can be obtained from quotients of Artin's braid group. ${ }^{14}$ The objects of the category are sequences of idempotents of the braid algebras. Morphisms $\Psi_{X, Y}, b_{X}$ are given by the images of the cabled versions of braids associated with them in the graphical calculus.

To give a precise description we introduce $\widetilde{\mathrm{EB}}_{k, l}$ to be the free module (over some ring) generated by the B-type tangles between $k$ upper and $l$ lower points. They may surround the fixed strand but are not allowed to touch it. We describe the construction in the cylinder picture only. The category $E B$ has as objects the numbers $\mathbb{N}_{0}$ and as morphisms the sets $\widetilde{\mathrm{EB}}_{k, l}$. Composition $f g$ is given as usual by putting $g$ on top of $f$. The tensor product $f \otimes g$ is obtained by replacing the fixed line of $g$ by $f$. The resulting category is a Coxeter-B braided category over the category of A-type (ordinary) tangles. The morphism $b_{n}$ is given by the picture of $b_{X}$ in Fig. 3 where the single line is replaced by a $n$ cable. It is tempting to introduce a projection $\pi$ as the map induced by deleting the fixed string. However, this will in general only be a morphism up to some scalar factors. The inclusion $i$ is given by adding a fixed string at the left.

In a next step one may impose skein relations to cut down the morphism spaces to finite dimension. A semi-simple category may then be obtained by taking as objects finite sequences of idempotents in the endomorphism algebras. If these algebras posess tensor representations one may also easily construct a functor to the vector spaces and hence reconstruct at least a restricted Coxeter-B braided Hopf algebra.

We now specialize to the case of the Temperley-Lieb category. 
Definition 5: The Temperley-Lieb Algebra $\mathrm{TB}_{n}$ of Coxeter type $B$ over a ring with parameters $c, d$ is generated by $e_{0}, e_{1}, \ldots, e_{n-1}$ and relations

$$
\begin{gathered}
e_{1} e_{0} e_{1}=c e_{1}, \\
e_{i} e_{j} e_{i}=e_{i} \quad|i-j|=1, i, j \geqslant 1, \\
e_{i} e_{j}=e_{j} e_{i} \quad|i-j|>1, \\
e_{0}^{2}=c e_{0}, \\
e_{i}^{2}=d e_{i} .
\end{gathered}
$$

This algebra has been introduced and studied by T. tom Dieck in Ref. 1. From this work we also recall that $\mathrm{TB}_{n}$ is semi-simple for generic parameters. It has dimension $\left(\begin{array}{l}2 n \\ n\end{array}\right)$ and $n+1$ simple components. The Bratteli diagram is given by Pascal's triangle. There are two series of JonesWenzl idempotents recursively defined by $f_{0}:=1+([3]-1)^{-1} e_{0}, g_{0}:=1-f_{0}, f_{m}:=f_{m-1}$ $+[2 m-1][2 m+1]^{-1} f_{m-1} e_{m} f_{m-1}, g_{m}:=g_{m-1}+[2 m-3][2 m-1]^{-1} g_{m-1} e_{m} g_{m-1}$. They satisfy $e_{i} f_{m}=f_{m} e_{i}=0=e_{i} g_{m}=g_{m} e_{i}=0=f_{m} g_{m} \forall i \leqslant m$. Furthermore, there is a Markov trace on $\mathrm{TB}_{n}$ that gives rise to a Jones polynomial of B-type. Alternatively it may be calculated from a B-type Kauffman bracket. ${ }^{1}$

Using these facts we can divide the morphisms spaces of $E B$ by the skein relations given by the B-type Kauffman bracket to obtain a category $\mathscr{S} B$. It is a Coxeter-B braided category over Turaev's $\mathscr{S} \cdot{ }^{14} \mathrm{~A}$ semi-simple category $\mathscr{T} B$ is then obtained in just the same way as in Ref. 14 . We defer the investigation of the question of whether this category is quasimodular to a subsequent paper.

We now construct tensor representations of $\mathrm{TB}_{n}$. Let $V$ be a vector space with basis $v^{i}$ and $B$ be any nondegenerate form on $V \otimes V$. We denote by $B_{i j}$ the matrix of $B$ and by $B^{i j}$ its inverse. Then the matrix $E_{i j}^{k l}:=B_{i j} B^{k l}$ defines a map $V \otimes V \rightarrow V \otimes V$. We obtain a tensor representation of $\mathrm{TB}_{n}$ on $V^{\otimes n}$ by representing $e_{i}$ as $E$ acting on tensor product spaces $i, i+1$. The parameter is $d$ $=\Sigma_{i, j} B_{i j} B^{k l}$. The generator $e_{0}$ acts as $F \otimes \mathrm{id} \otimes \cdots$, where $F:=c\left(B^{t}\right)^{-1} B$. The proof is a straightforward computation. Tensor representation of $\mathrm{TB}_{n}$ associated with the quantum group of $\mathrm{sl}_{2}$ where investigated in Ref. 1.

It is obvious that any tensor representation of $\mathrm{TB}_{n}$ defines a restricted tensor functor on the B-braided category in the sense of Proposition 4.

\section{QUANTUM FIELD THEORY ON THE HALF PLANE}

Consider a quantum field theory ${ }^{15}$ specified by a net of local observables $\mathscr{C}(\mathcal{O})$ living on the half plane $\left\{\left(x_{1}, x_{2}\right) \in \mathbb{R}^{2} \mid x_{1} \geqslant 0\right\}$. We assume that boundary conditions are imposed in such a way that we have reflection at the line $(0, \mathbb{R})$ by letting the full translation group $\mathbb{R}^{2}$ act on the half plane. This action is not free and this will lead to global morphisms and hence to the occurrence of a Coxeter-B braided tensor category.

Fields shall be localized in double cones. Here, we extend the usual notion of a double cone to include all translations of double cones. Thus we also have regions like those in the left of Fig. 4. This figure also shows the causal complement $\mathscr{O}^{\prime}$ of a double cone. A double cone that does not touch the boundary shall be called regular. Further we assume isotony and locality and the existence of a vacuum representation $\pi_{0}$ which is translation invariant and faithful for all local algebras $\mathscr{b}(\mathscr{O})$ of regular double cones $\mathscr{O}$. Note that in this setup the net $\mathscr{b}(\mathscr{Q})$ is directed so that there exists the inductive limit $\mathscr{C}$.

Now, let $\mathscr{O}_{1}, \mathscr{O}_{2}$ be two causally disjoint double cones of equal size as shown in the right half of Fig. 4. Further, let $\rho_{1}$ be a transportable morphism localized in $\mathscr{O}_{1}$ and let $\rho_{2} \sim \rho_{1}$ be localized 

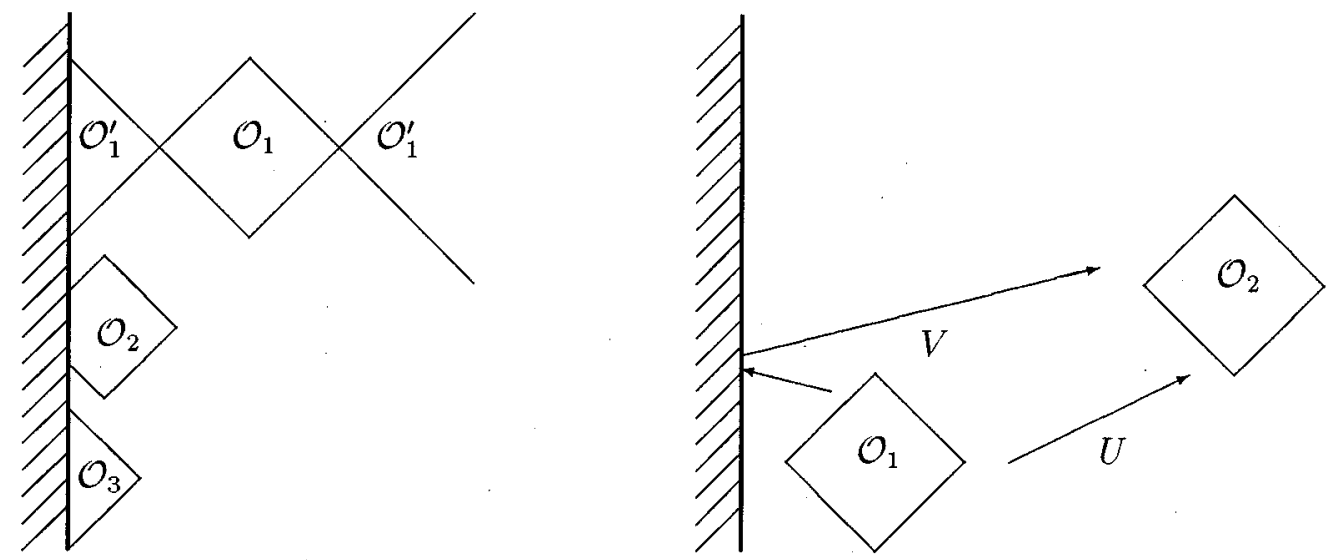

FIG. 4. The half plane with various double cones (on the left) and with the reflecting transportation that leads to global intertwiners (on the right).

in $\mathscr{O}_{2}$. Assume that $\rho_{1}$ (and thus $\rho_{2}$ ) is irreducible in the sense that $\pi_{0}{ }^{\circ} \rho_{1}$ is an irreducible representation of $\mathscr{C}$. There are two translations that map $\mathscr{O}_{1}$ onto $\mathscr{O}_{2}$ : A direct one and one that passes the reflecting boundary. Thus we have two charge transporters $U, V \in \operatorname{Mor}^{(G)}\left(\rho_{1}, \rho_{2}\right)$ : $=\left\{T \in \mathscr{A} \mid T \rho_{1}(A)=\rho_{2}(A) T \forall A \in \mathscr{A}\right\}$. (Later on, we will occasionally denote them by $U_{\rho_{1}}, V_{\rho_{1}}$ to make their dependence on the morphism clear.) Hence we have a self-intertwiner $Y:=U^{-1} V$ $\in \operatorname{Mor}^{(G)}\left(\rho_{1}, \rho_{1}\right)$. However, since $\rho_{1}$ is irreducible, we have (up to a phase which we absorb in the definition of $V) \pi_{0}(Y)=1$. We see that the vacuum representation may not be faithful in the presence of a boundary.

The localized and transportable morphisms form a Coxeter-B braided tensor category with projection. We have already given the set of global morphisms. Local morphisms are $\operatorname{Mor}\left(\rho_{1}, \rho_{2}\right):=\left\{T \in \mathscr{A} \mid \exists \mathcal{O}\right.$ regular, $\left.T \in \mathscr{A}(\mathcal{O}), \pi_{0}(T) \pi_{0}\left(\rho_{1}(A)\right)=\pi_{0}\left(\rho_{2}(A)\right) \pi_{0}(T) \forall A \in \mathscr{C}\right\}$. The localized transportable morphisms form a BTC as shown in Refs. 16, 17. The projection $\pi: \operatorname{Mor}^{(G)} \rightarrow$ Mor is given by $\pi_{0}$. The inclusion $i$ :Mor ${ }^{(G)} \rightarrow$ Mor exists because we assumed $\pi_{0}$ to be faithful on local algebras of regular double cones. The global morphism $b_{\rho_{1}}$ is $Y$ given above. (Note that $Y$ is independent of the choice of $\mathscr{O}_{2}$ because an additional local charge transporter $W$ would cancel out.) Equation (8) holds: Let $T \in \operatorname{Mor}(\varrho, \rho)$ be a local morphism. If $T$ is an isomorphism one can set $V_{\varrho}:=V_{\rho} T, U_{\varrho}:=U_{\rho} T$ if one chooses $\mathcal{O}_{2}$ to be casually disjoint to the localization double cones of $\rho$ and $\varrho$. Then one has $T Y_{\varrho}=T\left(U_{\rho} T\right)^{-1}\left(V_{\rho} T\right)=U_{\rho}^{-1} V_{\rho} T=Y_{\rho} T$. If $T$ is not an isomorphism then one may do an image/kernel splitting (by use of semi-simplicity of the $C^{*}$ category) $\rho=\rho_{a} \oplus \rho_{b}, \varrho=\varrho_{a} \oplus \varrho_{b}$ in such a way that $\varrho_{a}$ and $\rho_{a}$ are isomorphic by means of an isomorphism $\hat{T}$. It is then obvious that all charge transporters split like $U_{\rho}=U_{\rho_{a}} \oplus U_{\rho_{b}}$. Further we denote by $P_{a}^{\varrho}, P_{a}^{\rho}$ the projection endomorphisms of the indicated objects. Then one has $T=P_{a}^{\rho} T P_{a}^{\varrho}=P_{a}^{\rho} \hat{T} P_{a}^{\varrho}$. Now we can calculate $T Y_{\varrho}=P_{a}^{\rho} \hat{T} P_{a}^{\varrho}\left(U_{\varrho_{a}} \oplus U_{\varrho_{b}}\right)^{-1}\left(V_{\varrho_{a}} \oplus V_{\varrho_{b}}\right)$ $=P_{a}^{\rho} \hat{T} P_{a}^{\varrho} P_{a}^{\varrho}\left(U_{\rho_{a}} \hat{T} \oplus U_{\varrho_{b}}\right)^{-1}\left(V_{\rho_{a}} \hat{T} \oplus V_{\varrho_{b}}\right)=P_{a}^{\rho} \hat{T} P_{a}^{\varrho}\left(\left(U_{\rho_{a}} \hat{T}\right)^{-1} \oplus 0\right)\left(V_{\rho_{a}} \hat{T} \oplus 0\right) P_{a}^{\varrho}=P_{a}^{\rho}\left(U_{\rho_{a}}^{-1} \oplus 0\right)$ $\times\left(V_{\rho_{a}} \hat{T} \oplus 0\right) P_{a}^{\varrho}=P_{a}^{\rho}\left(U_{\rho_{a}}^{-1} V_{\rho_{a}} \oplus 0\right) \hat{T} P_{a}^{\varrho}=Y_{\rho} T$.

To show (10) we take another morphism $\varrho$ localised in $\mathscr{O}_{1}$. Using our assumptions that $\mathscr{Q}_{1}$, $\mathscr{Q}_{2}$ are causally disjoint we may express the statistics operators $\epsilon_{\rho_{1}, \varrho}=\varrho\left(U^{-1}\right) U, \epsilon_{\varrho, \rho_{1}}$ $=V^{-1} \varrho(V)$. Thus $\varrho(Y)=\epsilon_{\rho_{1}, \varrho} Y \epsilon_{\varrho, \rho_{1}}$.

A full proof of (11) seems to be difficult in the general situation. However, we note that (11) 
is established if one can show that $b_{X \otimes Y}=L\left(b_{X} \otimes b_{Y}\right)$ with some local morphism $L$ : Applying $\pi$ and using (18) one obtains $L=\Psi^{-2}$. Thus it suffices to argue that the $b_{X}$ are unique up to local morphisms.

Similar results are obtained for QFT on the circle $S^{4} .{ }^{16}$ The most important common feature of these two topologies is that the action of the translation group is not free. Fredenhagen, Rehren, and Schroer already point out that the occurrence of global intertwiners is linked to the existence of a forbidden direction; a fact that can most clearly be seen in our setup.

${ }^{1}$ T. tom Dieck, "Knotentheorien und Wurzelsysteme I, II," Math. Gottingensis 21 (1993) and Math Gottingensis 44 (1993).

${ }^{2}$ T. tom Dieck, “'On tensor representations of knot algebras," Math. Gottingensis 45 (1995).

${ }^{3}$ S. Goshal and A. B. Zamolodchikov, "Boundary $S$ matrix and boundary state in two-dimensional integrable QFT, " Int. J. Mod. Phys. A 9, 3841 (1994).

${ }^{4}$ R. Häring-Oldenburg, "New solutions of the reflection equation derived from Type B BMW algebras," J. Phys. A 29 , 5945-5948 (1996).

${ }^{5}$ R. Häring-Oldenburg, "The Potts Model with a reflecting boundary," J. Knot Theory and its Ramifications (to appear).

${ }^{6}$ S. Lamborpoulou, "Solid torus links and Hecke algebras of B-type,"' in Proc. Quantum Topology, Kansas 1993, edited by D. Yetter (World Scientific, Singapore, 1994).

${ }^{7}$ R. Häring-Oldenburg, "The reduced Birman-Wenzl algebra of Coxeter Type B," (preprint).

${ }^{8}$ S. Majid, Foundations of Quantum Group Theory (Cambridge University Press, Cambridge, 1995).

${ }^{9}$ R. Häring-Oldenburg, "Reconstruction of weak quasi Hopf algebras," J. Algebra (to appear).

${ }^{10}$ T. tom Dieck and R. Häring-Oldenburg, "Tensor categories with cylinder braiding", (in preparation).

${ }^{11}$ S. Majid, "Solutions of the Yang-Baxter equations from Braided-Lie algebras and braided groups," J. Knot Theory and its Ramifications 4, 673-697 (1995).

${ }^{12}$ A. N. Kirillov and N. Reshetikhin, " $q$-Weyl group and a multiplicative formula for universal $R$-matrices," Commun. Math. Phys. 134, 421-431 (1990).

${ }^{13}$ T. tom Dieck and R. Häring-Oldenburg, "Quantum groups and cylinder braiding," Forum Math. (submitted).

${ }^{14}$ V. G. Turaev, Quantum Invariants of Knots and 3-manifolds (de Gruyter, Berlin, 1994).

${ }^{15}$ R. Haag, Local Quantum Physics (Springer, Berlin, 1992).

${ }^{16}$ K. Fredenhagen, K.-H. Rehren, and B. Schroer, "Superselection sectors with braid group statistics and exchange algebras I, II,’ Commun. Math. Phys. 125, 201-226 (1989); Rev. Math. Phys. Dec., 113-157 (1992).

${ }^{17}$ J. Fröhlich and T. Kerler, Quantum Groups, Quantum Categories and Quantum Field Theory, Springer Lecture Notes on Mathematics, 1542 (Springer, Berlin, 1993). 